\title{
Review Paper on Wireless Power Transmission
}

\author{
S. D. Rankhamb ${ }^{1}$, A. P. Mane ${ }^{2}$ \\ ${ }^{1}$ ME Student (E\&TC), T.P.C.T’s College of Engineering, Osmanabad-413501 \\ ${ }^{2}$ Professor, E\&TC Department, T.P.C.T’s College of Engineering, Osmanabad-413501
}

\begin{abstract}
In present era every person needs wireless system, but still power transmission for low power device we are using wired device continuous power supply is one of the major issues in the purpose of the application of wireless sensor network. But in the power system of wireless Sensor Network (WSN), the battery has a very limited lifetime and is not replaced yet by any other continuous power system. Imagine a future in which wireless power transfer is feasible: cell phones, household robots, mp3 players, laptop computers and other portable electronics capable of charging themselves without ever being plugged in therefore we present the concept of transmitting power without using wires i.e. transmitting power as microwaves from one place to another is in order to reduce the cost, transmission and distribution losses increase efficiency. Typical WPT is a point to-point power transmission. For the WPT, we had better concentrate power to receiver. It was proved that the power transmission efficiency can approach close to $100 \%$.
\end{abstract}

Keywords: Wireless sensor network, Nikola Tesla Experiment, Microwave power transmission (MPT), Wireless power transmission (WPT), Power Satellites.

\section{Introduction}

Wireless power transfer (WPT) is the transmission of electrical power from a power source to a consuming device without using discrete manmade conductors. Researchers have developed several techniques for moving electricity over long distance without wires. Some exist only as theories or prototypes but others are already in use. This paper provides the techniques used for wireless power transmission. It is a generic term that refers to a number of different power transmission technologies that use timevarying electromagnetic fields.

Wireless transmission is useful to power electrical devices in case where interconnecting wires are inconvenient, hazardous, or are not possible. For example the life of WSN is its node which consist of several device controllers, memory, sensors, actuators, transceivers and battery and battery. The transceiver can operate in four states, i.e 1) Transmit 2) Receive 3) Idle and 4) Sleep. The major energy problem of a transmitter of a node is its receiving in idle state, as in this state it is always being ready to receive, consuming great amount of power.

However, the batter has a very short lifetime and moreover in some developments owing to both practically and economically infeasible or may involve significant resists to human life. That is why energy harvesting for WSN in replacement of battery is the only and unique solution. In wireless power transfer, a transmitter device source, such as the mains power line, transmits power by electromagnetic fields across an intervening space to one or more receiver devices, where it is converted back to electric power and utilized. In communication the goal is the transmission of information, so the amount of power reaching the receiver is unimportant as long as it is enough that signal to noise ratio is high enough that the information can be received intelligibly. In wireless communication technologies, generally, only tiny amounts of power reach the receiver. By contrast, in wireless power, the amount of power received is the important thing, so the efficiency (fraction of transmitted power that is received) is the more significant parameter.

\subsection{Issues in WPT}

One of the major issue in power system is the losses occurs during the transmission and distribution of electrical power. As the demand increases day by day, the power generation increases and the power loss is also increased. The major amount of power loss occurs during transmission and distribution. The percentage of loss of power during the transmission and distribution is approximated as $26 \%$ [4]. The main reason for power loss during transmission and distribution is the resistance of wires used for grid. The efficiency of power transmission can be improved to certain level by using high strength composite over head conductors and underground cables that use high temperature super conductor. But, the transmission is still inefficient. According to World Resources Institution (WRI), India's electricity grid has the highest transmission and distribution losses in the world a whopping27\%.Numbers published by various Indian government agencies put that number at $30 \%, 40 \%$ and greater than $40 \%$. This is attributed to technical losses (grid's inefficiencies) and theft [4]. The above discussed problem can be solved by choose an alternative option for power transmission which could provide much higher efficiency, low transmission cost and avoid power theft. Microwave Power Transmission is one of the promising technologies and may be the righteous alternative for efficient power transmission.

\subsection{Field Regions}

Electric and magnetic fields are created by charge particles in matter such as electrons. A stationary charge creates an electrostatic field in the space around it. A steady current of charge (direct current, DC) creates a static magnetic field around it. The above fields contain energy, but cannot carry power because they are static. However time-varying fields can carry power. Accelerating electric charge, such as are found in an alternating current (AC) of electrons in a wire, create time-varying electric and magnetic fields in the space around them. These fields can exert oscillating force on the electrons in a receiving "antenna", causing them to move back and forth. These represent alternating current which 


\section{International Journal of Science and Research (IJSR) \\ ISSN (Online): 2319-7064 \\ Index Copernicus Value (2013): 6.14 | Impact Factor (2014): 5.611}

can be used to power a load. The oscillating electric and magnetic fields surrounding moving electric charges in an antenna device can be divided into two regions, depending on distance. Drange from the antenna[10]. Different technologies are used for transmitting power: Near-field or non-radiative region- This means the area within about wavelength $(\lambda)$ of the antenna.

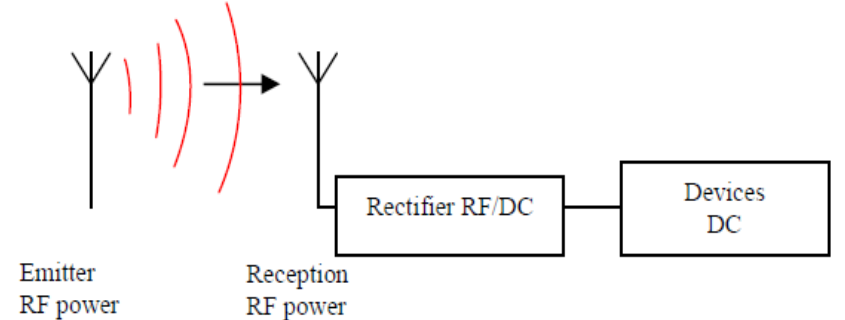

Figure 1.1: Schematic view of the WPT system

In this region the oscillating electric and magnetic fields are separate and power can be transferred via electric fields by capacitive coupling (electrostatic induction) between metal electrodes, or via magnetic fields by inductive coupling(electromagnetic induction) between coils of wire[5][6]. The range of these fields is short, and depends on the size and shape of the "antenna" devices, which are usually coils of wire. The fields, and thus the power transmitted decrease exponentially with distance, so if the distance between the two "antennas" Drange is much larger than the diameter of the "antenna" Dant very little power will be received.Therefore,these techniques cannot be used for long distance power transmission.

Resonance, such as resonant inductive coupling, can increase the coupling, can increase the coupling between the antennas greatly, allowing efficient transmission at somewhat greater distance, although the fields still decrease, although the fields still decrease exponentially. Therefore the range of near-fields devices is conventionally divided into two categories: Short range- up to about one antenna diameter: Drange $\leq$ Dant.This is the range over which ordinary non-resonant capacitive or inductive coupling can transfer particle amounts of power.Mid-range-up to 10 times the antenna diameter: range $\leq 10$ Dant.This is range over which ordinary non-resonant capacitive or inductive coupling can transfer practical amount of power.

Far-field or radiative region: Beyond about 1 wavelength $(\lambda)$ of antenna, the electric and magnetic fields perpendicular to each other and propagate as an electromagnetic wave; example are radio waves,microwave,or light waves. This part of the energy is radiative, meaning it leaves the antenna whether or not there is a receiver absorbs it. The portion of energy which does not strike the receiving antenna is dissipated and lost to the system. The amount of power emitted as electromagnetic waves by an antenna depends on the ratio of the antenna's size Dant to the wavelength of waves $\lambda$,which is determined by the frequency $f$ where the frequency: $\lambda=\mathrm{c} / \mathrm{f}$. At low frequencies $\mathrm{f}$ where the antenna is much smaller than the size of the waves, Dant $<<\lambda$, very little power is radiated. Therefore the near-field devices above, which use lower frequencies, radiate almost none of their energy as electromagnetic radiation. Antennas about the same size as the wavelength Dant $\approx \lambda$ such as monopole or dipole antennas radiate power efficiently, but the electromagnetic waves are radiated in all directions. so if the receiving antenna is far away, only a small amount of the radiation will hit it.Therefore,these can be used for shorter range inefficient power transmission but not for short range transmission but for long range transmission. However, unlike fields, electromagnetic radiation can be focused by reflection or refraction into beams. By using a high gain antenna or optical system which concentrates the radiation into a narrow beam aimed at the receiver, it can be used for long range power transmission. From the Rayleigh criterion, to produce the necessary to focus a significant amount of the energy on a distant receiver, an antenna must be much larger than the wavelength of the wave used Dant $>\lambda=\mathrm{c} / \mathrm{f}$. Practical beam power devices require wavelength in the centimeter region or below in the corresponding to frequencies above $1 \mathrm{GHz}$, in the microwave range.

\subsection{Classification of WPT}

\subsubsection{Non- radiative (Near -field techniques)}

In near-field or non-radiative techniques, power is transferred over short distance by magnetic fields using inductive coupling between coils of wire or in a few devices by electric fields using capacitive coupling between electrode [5][8].

A current focus is to develop wireless systems to charge mobile and handheld computing devices such as cellphones, digital music players and portable computers without being tethered to a wall plug. Application of this type are electric toothbrush chargers, RFID tags, smartcards and chargers for implantable medical devices like artificial cardiac pacemaker, and inductive powering or charging of electric vehicles like trains or buses. Fig .1.2 shows the classification of WPT.

\subsubsection{Radiative (Far-field techniques)}

In radiative or Far-field techniques, also called power beaming, power is transmitted by beams of electromagnetic radiation, like microwaves or laser beams. These techniques can transport energy longer distances but must be aimed at the receiver. Proposed application for this type is solar power satellites, and wireless powered drone aircraft.

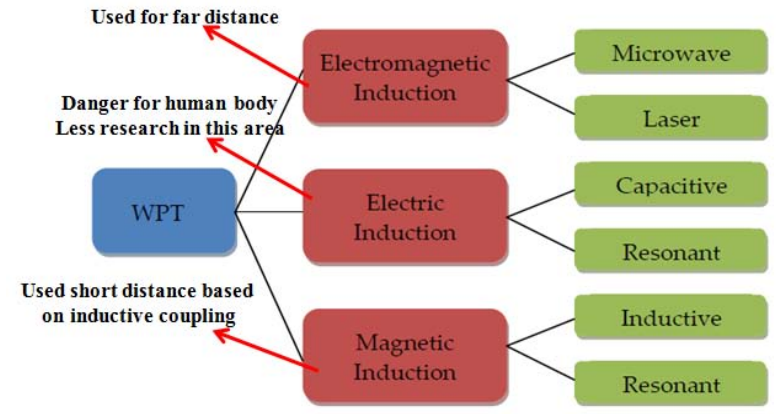

Figure 1.2: Classification of wireless power transmission.

\section{Literature Review}

\subsection{History}

In 1826 Andre-Marie Ampere developed ampere's circuital law showing that electric current produces a magnetic 


\section{International Journal of Science and Research (IJSR) \\ ISSN (Online): 2319-7064 \\ Index Copernicus Value (2013): 6.14 | Impact Factor (2014): 5.611}

field.Michael Faraday developed Faraday's law of induction in 1831, describing the electromagnetic force induced in a conductor by a time-varying magnetic flux. In 1862 James Clerk Maxwell synthesized these and other observations, experiments and equations of electricity, magnetism and optics into a consistent theory, deriving Maxwell's equations. This set of partial differential equations forms the basis for modern electromagnetic including the wireless transmission of electrical energy.

\subsubsection{Tesla's Experiment}

Tesla demonstrating wireless power transmission in a lecture at Columbia College, New York, in 1891.The two metal sheets are connected to his Tesla coil oscillator, which applies a high frequency oscillating voltage. The oscillating electric fields between the sheets ionizes the low pressure gas in the two long Geissler tubes he is holding, causing them to glow by fluorescence, similar to neon lights. Experiment in resonant inductive transfer by Tesla at Colorado Springs 1899.The coil is in resonance with Tesla's magnifying transmitter nearby, powering the light bulb at bottom. Inventor Nikola Tesla performed the first experiments in wireless power transmission in wireless power transmission at the turn of the $20^{\text {th }}$ century, and may have done more to popularize the idea than any other individual. In the period 1891 to 1904 he experimented with transmitting power by inductive and capacitive coupling using spark-excited radio frequency resonant transformer, now Called Tesla coils, which generated high AC voltages. With these he was able to transmit power for short distances without wires. In demonstrations before the American Institute of Electrical Engineers and the 1893 Columbian Exposition in Chicago he lit light bulbs from across a stage.He found he could increase the distance by using a receiving LC circuit tuned to resonance with the transmitter's LC circuit, using resonant inductive coupling. At his Colorado springs laboratory during 1899-1900,by using voltages of the order of 10 megavolts generated by an enormous coil. he was able to light three incandescent lamps at a distance of a about one hundred feet. The resonant inductive coupling which Tesla pioneered is now a familiar technology used throughout electronics and is currently being widely applied to short-range wireless power systems[1][2].

\subsection{Wireless Power Transmission System}

W.C.Brown, the pioneer in wireless power transmission technology, has designed, developed a unit and demonstrated to show how power can be transferred through free space by microwave. The concept of wireless power transmission system is explained with functional block diagram shown in Fig.2.1

In the transmission side,the microwave power source generates microwave power and the output power is controlled by electronic control circuits.

The waveguide ferrite circulator which protects microwave source from reflected power is connected with the microwave power source through the coax-waveguide adaptor. The tuner matches the impedance between the transmitting antenna and the microwave source. The attenuated signals will be then separated based on the direction of signal propagation by Directional Couplers by Directional Coupler. The transmitting antenna radiates the power uniformly through free space to the rectenna. In the receiving side, a rectenna receives the transmitted power and converts the microwave power into DC power.

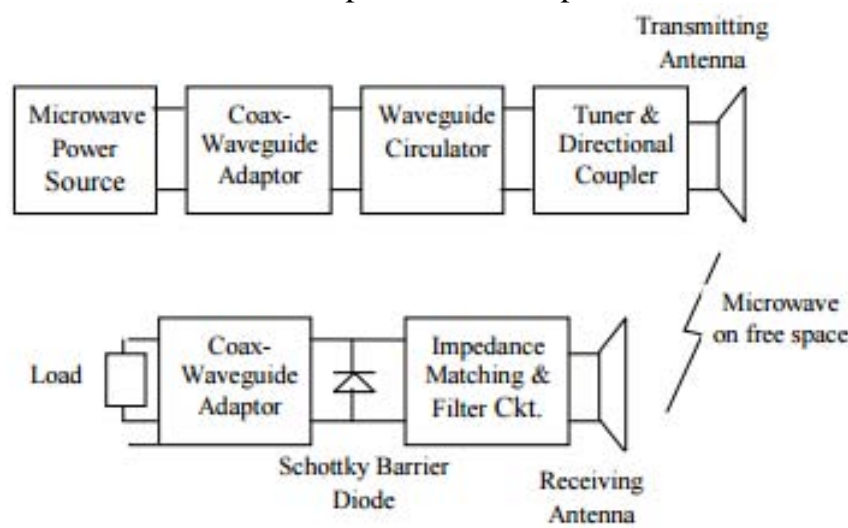

Figure 2.1: Functional Block Diagram of Wireless Power Transmission System.

The impedance matching circuit and filter is provided to setting the output impedance of a signal source equal to the rectifying circuit. the rectifying circuit consists of schottky barrier diodes converts the received microwave power into DC power[3].

\subsection{Energy Harvesting}

In the context of wireless power, energy harvesting, also called power harvesting or energy scavenging, is the conversion of ambient energy from the environment to electric power, mainly to power small autonomous wireless electronic devices. The ambient energy may come from stray electric power, mainly to power small autonomous wireless electronic devices. The ambient energy may come from stray electric or magnetic fields or radio waves from nearby electrical equipment, light, thermal energy (heat),or kinetic energy such as vibration or motion of the device.

Although the effiency of conversion is usually low and the power gathered often minuscule (mill watts or microwatts),it can be adequate to run or recharge small micro power wireless devices such as remote sensors, which are proliferating in many fields. This new technology is being developed to eliminate the need for battery replacement or charging of such wireless devices, allowing them to operate completely autonomously.

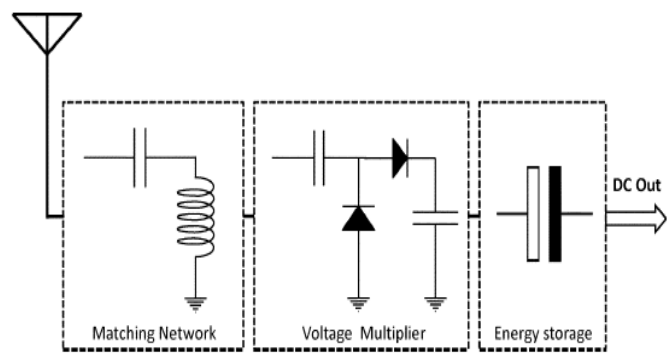

Figure 2.2: Energy Harvesting Circuit 


\section{Advantages, Disadvantages \& Biological Impacts of WPT}

\section{Advantages}

- It makes devices more convenient and thus more desirable to purchasers, by eliminating the need for a power cord or battery replacement.

- The power failure due to short circuit and fault on cables would never exist in transmission.

- Reduction of E-waste by eliminating the need of power cords.

- Wireless charging offers no corrosion as the electronics are all enclosed, away from water or oxygen in the atmosphere [2].

\section{Disadvantages}

- The capital cost for particle implementation of WPT seems very high.

- WPT may cause interference with present communication systems.

- Less efficiency compared to traditional charging.

\section{Biological Impacts}

Common beliefs fear the effect of microwave radiation. But the studies proven that the microwave radiation level would be never higher than the dose received while opening the microwave oven door, meaning it slightly higher.

\section{Application of WPT}

- Moving targets such as fuel free airplanes, fuel free electric vehicles, moving robots and fuel free rackets.

- Automatic wireless charging for mobile robots, cordless tools and instrument which eliminates complex mechanisms, and labour intensive manual recharging and battery replacement.

- Another application of WPT are solar power satellites, energy to remote areas, broadcast energy globally.

- WPT are used for Ubiquitous power source, RF power Adaptive Rectifying Circuits (PARC).

\section{Future Scope}

Witricity is building a near field wireless charging apparatus for consumer devices with the help of the Haier group, a chinese electronics manufacturer. Witricity demonstrated this technology by wireless powering a 32 inch television at a distance of six feet. Delphi Automotives is working with Witricity to develop a wireless charging system for electric cars. The groundbreaking technology will enable to automotive manufacturer to integrate wireless charging into the design of hybrid \& electric vehicles. There is another standard protocol for charging mobile phone initiated by the Wireless Power Consortium [9].

\section{Conclusion}

The concept of wireless power transmission offers greater possibilities for transmitting power with negligible losses. In the long run, this could reduce our society's dependence on batteries, which are currently heavy and expensive. As wireless technology is getting popular now a days, the demand of battery is also decreasing. For the long range power transmission power can be sent from source to receivers instantaneously without wires, reducing the cost. Batteries need to be recharge or changed eventually, hence the need for this kind of work.

\section{References}

[1] Nikola Tesla,"The Transmission of Electrical Energy Without Wires as a Means for Furthering Peace," Electrical World and Engineer. Jan.7, p. 21, 1905.

[2] Inductive charging, Wireless charging homepage on Wikipedia [online] Available: http://en.wikipedia.org/wiki/Inductive_charging

[3] W.C Brown, J.R Mims and N.I Heenan, "An Experimental Microwave-Powered Helicopter" 965 IEEE International Convention Record Vo. 13, Part 5, Pp. 225-235.

[4] http://cleantechindia.wordpress.com/2008/07/16/indiase leletricity-transmission-and-distribution-losses/

[5] Farouk Balouchi and Bob Gohn, "Wireless Power: Mobile Devices, Consumer Electronics, Industrial Devices, Wireless Power Infrastructure, and Wireless Charging of Electric Vehicles: Technology Analysis, Environmental Impact, and Market Forecasts," Pike Research Report, Published 2Q 2012.

[6] Gopinath, Ashwin (August 2013)."All about Tranferring Power Wirelessly" (PDF). Electronics For You E-zine(EFY Enterprises Pvt.Ltd) Retrieved January 16,2015

[7] Tomar,Anuradha;Gupta,Sunil(July2012).

"WirelessPowerTransmission:Application and Components" International Journal of Engineering Research \&Technology

[8] http://www.hometoys.com/content.php?post_type=1958

[9] TheWitricitywebsite[online].Available:http://www.witri city.com/pages/companyhtml

[10] https://en.m.wikipedia.org/wiki/wireless_power_transfe r 